\title{
Study the exchange bias field in ferromagnetic film on antiferromagnetic substrate
}

\author{
S. V. Belim \\ sbelim@mail.ru \\ Omsk State Technical University, Omsk, 644050, Russia \\ Siberian State Automobile and Highway University, Omsk, 644080, Russia
}

\begin{abstract}
In the article, exchange bias in a ferromagnetic film on an antiferromagnetic substrate is studied. These systems are widely used in spintronics devices to fix the state of one of the ferromagnets. The computer simulation method is used for the study. The Ising model and Metropolis algorithm are used. A thin ferromagnetic film on a semi-infinite antiferromagnetic substrate is considered. The layered antiferromagnet model is used for the substrate. The exchange value for the antiferromagnet is lower than for the ferromagnetic film. The Neel temperature for the substrate is lower than the Curie temperature for the ferromagnetic film. For both components of the system, phase transition temperatures are calculated. The exchange bias field is created by exchange interaction at the interface of the film with the substrate. At temperatures above the Neel temperature, the surface layer for the antiferromagnet is not compensated and does not create exchange bias. The dependence of the exchange bias field on the system's temperature is investigated. Exchange bias shifts the hysteresis loop. The position of the hysteresis loop center determines the exchange bias field. As the temperature decreases, the chess magnetization for the antiferromagnetic substrate and the magnetic moment for the boundary spins layer increase. A computer experiment was performed. The dependence of the exchange bias field on temperature near the phase transition point for the antiferromagnet is linear. As the temperature decreases, the exchange bias becomes constant. This transition is associated with maximizing the chess magnetization of the antiferromagnetic substrate. With a decrease in temperature, the width of the hysteresis loop increases. The width of the hysteresis loop decreases linearly with increasing temperature. A comparison is made with the results of real experiments.
\end{abstract}

Keywords: multilayer systems, exchange bias, ferromagnetic films, Ising model.

УДК: 537.622 .5

\section{Изучение поля обменного смещения в ферромагнитной пленке на антиферромагнитной подложке}

\author{
Белим С. В. \\ Омский государственный технический университет, Омск, 644050, Россия \\ Сибирский государственный автомобильно-дорожный университет, Омск, 644080, Россия
}

В статье проведено исследование обменного подмагничивания в ферромагнитной пленке на антиферромагнитной подложке. Эти системы широко используются в устройствах спинтроники для закрепления состояния одного из ферромагнетиков. Для исследования использован метод компьютерного моделирования. Использованы модель Изинга и алгоритм Метрополиса. Рассмотрен случай тонкой ферромагнитной пленки на полубесконечной антиферромагнитной подложке. Рассмотрен случай слоистого антиферромагнетика. Величина обменного взаимодействия для антиферромагнетика ниже, чем для ферромагнитной пленки. Вследствие этого температура Нееля подложки ниже температуры Кюри ферромагнитной пленки. Для обеих составляющих системы определены температуры фазовых переходов. Поле обменного подмагничивания создается обменным взаимодействием на границе соприкосновения пленки с подложкой. При температурах выше температуры Нееля поверхностных слой антиферромагнетика не является компенсированным и не создает обменного подмагничивания. Исследована зависимость поля обменного подмагничивания от температуры системы. Обменное подмагничивание вызывает 
смещение петли гистерезиса. Положение центра петли гистерезиса определяет величину поля обменного подмагничивания. С понижением температуры происходит рост шахматной намагниченности антиферромагнитной подложки и магнитного момента пограничного слоя спинов. Проведен компьютерный эксперимент. Показано, что зависимость поля обменного подмагничивания от температуры вблизи точки фазового перехода антиферромагнетика линейная. При дальнейшем понижении температуры происходит переход на постоянную величину обменного подмагничивания. Этот переход связан с достижением максимума шахматной намагниченности антиферромагнитной подложки. Показано, что при понижении температуры происходит увеличение ширины петли гистерезиса. Зависимость ширины петли гистерезиса от температуры также является линейной. Проведено сравнение с результатами реальных экспериментов.

Ключевые слова: двухслойные системы, обменное подмагничивание, ферромагнитные пленки, модель Изинга.

\section{1. Введение}

Под обменным смещением понимается влияние одной магнитной среды на величину намагниченности в другой магнитной среде за счет обменного взаимодействия на границе их соприкосновения. Обменное смещение возникает при контакте двух разных магнитных сред $[1,2]$. Наибольший интерес представляет исследование обменного смещения в бислойной системе, состоящей из ферромагнитной (FM) и антиферромагнитной (AFM) пленок. Такие бислойные системы применяются в устройствах спинтроники для закрепления состояния одного из ферромагнитных слоев [2]. Составляющие бислойной системы подбираются таким образом, чтобы температура Кюри $\left(T_{C}\right)$ ферромагнитного материала была больше температуры Нееля $\left(T_{N}\right)$ антиферромагнитного материала. В бислойной системе пленки магнитно связаны обменным взаимодействием. Эта обменная связь влияет на явление гистерезиса. При охлаждении ниже $T_{N}$ во внешнем магнитном поле происходит три изменения петли гистерезиса по сравнению с одиночной ферромагнитной пленкой. Во-первых, происходит смещение петли вдоль оси индукции магнитного поля. Новый центр петли определяет магнитное поле обменного смещения $h_{E B}$. Этот сдвиг обусловлен обменным взаимодействием на границе пленок. При этом существует температура блокировки $T_{B}\left(T_{B}<T_{N}\right)$, выше которой поле обменного смещения становится нулевым и восстанавливается петля гистерезиса изолированного ферромагнетика. Во-вторых, изменяется коэрцитивное поле, что приводит к увеличению ширины петли гистерезиса. В-третьих, может происходить деформация петли гистерезиса [1-4].

Для двухслойной системы FM/AFM явление обменного смещения определяется спиновой структурой на межфазных плоскостях. Если поверхность АFM имеет нулевую намагниченность, то она классифицируется как компенсированная. Наличие обменного смещения объясняется некомпенсированным состоянием граничной плоскости AFM [4]. Наличие некомпенсированных доменов является необходимым требованием для сдвига петли гистерезиса [5].

Важным моментом является значение обменной связи между различными пленками на границе соприкосновения. Модель, предложенная в работе [6], предсказывает линейную зависимость поля обменного смещения $h_{E B}$ от межфазного обменного интеграла $J_{E B}$ и обратную зависимость от толщины ферромагнитной пленки $D_{F M}$ :

$$
h_{E B} \sim \frac{J_{E B}}{D_{F M}} .
$$

В рамках феноменологической модели [7] было предсказано наличие насыщения для поля обменного взаимодействия с ростом межфазного обменного взаимодействия.

Вопрос влияния обменного взаимодействия на границе двух магнетиков активно исследуется методом компьютерного моделирования [8-17]. В этих работах были подтверждены результаты о линейной зависимости обменного смещения от величины обменного взаимодействия на границе пленок. Нелинейная зависимость для больших значений межфазного обменного интеграла была получена в работе [18].

Все эти результаты верны при полностью скомпенсированном пограничном слое антиферромагнетика. В этом состоянии в приграничной плоскости антиферромагнетика все спины ориентированы одинаково. Скомпенсированный слой образуется при температурах существенно ниже температуры Нееля. Тем не менее интерес представляет зависимость поля подмагничивания от температуры вблизи точки фазового перехода.

Данная статья посвящена исследованию влияния температуры бислойной системы на величину поля обменного смещения в ферромагнитной пленке методом компьютерного моделирования.

\section{2. Описание системы}

Рассмотрим систему, состоящую из ферромагнитной пленки (FM) и полубесконечного антиферромагнетика $(\mathrm{AFM})$. В реальных экспериментах и при создании устройств спинтроники толщина антиферромагнетика значительно больше толщины ферромагнитной пленки. Такое соотношение выбирается, чтобы избежать влияния ферромагнетика на антиферромагнетик. Ферромагнитная пленка ниже точки Кюри создает магнитное поле, которое может приводить к разрушению антиферромагнетизма. Если толщина ферромагнитной пленки много меньше толщины антиферромагнитной пленки, то влияние будет незначительным. Для того чтобы исключить данное влияние в компьютерном эксперименте рассматривалась ферромагнитная пленка на полу- 
бесконечной антиферромагнитной подложке. FM пленка имела толщину $D$. При компьютерном моделировании толщина пленки измеряется количеством слоев спинов. Введем оси координат таким образом, чтобы пленка и поверхность антиферромагнетика были параллельны плоскости ОXY. Будем рассматривать систему в рамках модели Изинга [19], в котором каждый спин может находится в одном из двух состояний $(S=1 / 2$ или $S=-1 / 2)$. Взаимодействие спинов в различных слоях описывается разными обменными интегралами. В ферромагнитном слое $(0 \leq z<D)$ значение обменного интеграла $J$, в антиферромагнитном слое $(D \leq z)$ обменный интеграл $J_{a}$. При этом использовалась модель слоистого ферромагнетика. Для спинов, находящихся в одном слое энергетически выгодно упорядочиваться в одном направлении. Для спинов в соседних слоях энергетически выгодно упорядочиваться в противоположных направлениях. Температура фазового перехода прямо пропорциональная величине обменного интеграла. Мы рассматриваем бислойную систему, для которой температура Кюри ферромагнитной пленки превышает температуру Нееля антиферромагнитной пленки $\left(T_{C}>T_{N}\right)$, поэтому будет выполняться неравенство $J \geq J_{a}$. Кроме этого, происходит взаимодействие спинов на границе пленок. Это взаимодействие носит обменный ферромагнитный характер и определяется обменным интегралом $J_{E B}$.

Гамильтониан такой системы во внешнем магнитном поле запишется в виде:

$$
H=-J \sum_{0 \leq z<D} S_{i} S_{j}+J_{a} \sum_{D \leq z}(-1)^{\sigma} S_{i} S_{j}-J_{E B} \sum_{z=D} S_{i} S_{j}+\mu h_{0} \sum S_{i} .
$$

Здесь $S_{i}-$ спин в $i$-ом узле, $h_{0}$ - напряженность магнитного поля, $\mu$ - магнетон Бора, $\sigma-$ параметр равный 0 , если спины в одной плоскости, и равный 1 , если спины в соседних плоскостях, $J_{E B}-$ обменный интеграл взаимодействия спинов на границе взаимодействия ферромагнетика и антиферромагнетика. Во всех слагаемых суммирование осуществляется только по ближайшим соседям. В третьем слагаемом один спин берется из FM пленки, а второй из AFM пленки.

Перейдем к безразмерным относительным величинам:

$$
R_{a}=J_{a} / J, R_{E B}=J_{E B} / J, h=\mu h_{0} / J .
$$

Запишем гамильтониан через относительные величины.

$$
H / J=-\sum_{0 \leq z<D} S_{i} S_{j}+R_{a} \sum_{D \leq z}(-1)^{\sigma} S_{i} S_{j}-R_{E B} \sum_{z=D} S_{i} S_{j}+h \sum S_{i} .
$$

Для температуры системы $t$ будем использовать величину, измеряемую в единицах обменного интеграла ферромагнитной системы $T=k t / J$, где $k-$ постоянная Больцмана.

Намагниченность FM пленки вычислялась как суммарное значение спина на одну частицу.

$$
m=\sum_{i=1}^{N} S_{i} / N
$$

$N$ - количество спинов в FM пленке.
Для AFM пленки вычислялось значение разности намагниченностей четных и нечетных слоев спинов.

$$
m_{a}=\left(\sum_{\text {even }} S_{i}-\sum_{\text {odd }} S_{i}\right) / N .
$$

Компьютерное моделирование осуществлялось для ферромагнитных пленок с линейными размерами $L \times L \times D$. Вдоль направлений осей $O X$ и $O Y$ использовались периодические граничные условия. Для моделирования полубесконечной антиферромагнитной подложки использовались системы с линейным размером $L \times L \times 2 L$. Компьютерное моделирование осуществлялось с помощью алгоритма Метрополиса [19]. Кластерные Вольфа [20] и Свендсена-Янга [21] не позволяют получить петлю гистерезиса, так как полностью стирают информацию о предыдущем состоянии системы.

Система исследовалась при температурах ниже температуры Кюри FM пленки. При этом выбирались температуры как выше, так и ниже температуры Нееля AFM подложки. Для определения температуры Кюри и температуры Нееля использовались куммулянты Биндера четвертого порядка [19]:

$$
U=1-\frac{\left\langle m^{4}\right\rangle}{3\left\langle m^{2}\right\rangle^{2}}, \quad U_{a}=1-\frac{\left\langle m_{a}^{4}\right\rangle}{3\left\langle m_{a}^{2}\right\rangle^{2}} .
$$

Угловые скобки использованы для обозначения термодинамического усреднения по состояниям системы. Температура фазового перехода определяется как точка пересечения графиков зависимости куммулянтов Биндера от температуры для систем различного линейного размера $L$ [19]. Температура Кюри $T_{C}$ вычислялась на основе куммулянтов $U$, а температура Нееля $T_{N}-$ на основе куммулянтов $U_{a}$.

Для моделирования петли гистерезиса система приводилась в равновесное состояние в магнитном поле $h_{\text {max }}$ с помощью алгоритма Метрополиса с числом шагов Монте-Карло $n$. После этого значение магнитного поля уменьшалось на $\Delta h$ и система приводилась в равновесие с числом шагов Монте-Карло равным $n_{1}$. Этот процесс продолжается до тех пор, пока значение внешнего магнитного поля не достигнет $-h_{\text {max. }}$ Затем на каждом шаге магнитное поле увеличивается на $\Delta h$ до достижения значения $h_{\text {max }}$. Число шагов Монте-Карло при переходе из одного состояния в другое $n_{1} \ll n$ для того, чтобы не терялась полностью информация о предыдущем состоянии. Петля гистерезиса строилась для различных температур $T<T_{C}$. Начальная температура была выше температуры Нееля $\left(T>T_{N}\right)$ для того, чтобы антиферромагнетик находился в парамагнитной фазе и не создавал поля обменного смещения. В дальнейшем температура понижалась и исследовалось смещение петли гистерезиса. По смещению центра петли гистерезиса определялось значение поля обменного смещения.

\section{3. Результаты компьютерного моделирования}

В компьютерном эксперименте использовались пленки с линейными размерами от $L=20$ до $L=36$ с шагом 
$\Delta L=4$. Данные размеры системы позволяют вычислить температуру фазового перехода с точностью до одного знака после запятой, что является приемлемым результатом в рамках выбранной модели. Дальнейшие расчеты намагниченности выполнялись для систем с линейным размером $L=36$. Ферромагнитная пленка имела постоянную толщину $D=4$. Исследования для пленок другой толщины не проводились, так как они могут быть вычислены из общей закономерности, полученной в работе [6]. Для отношения обменных интегралов антиферромагнетика и ферромагнетика было выбрано значение $R_{a}=0.6$. Важно, чтобы это отношение было меньше единицы, так как температура Нееля подложки должна быть ниже температуры Кюри пленки. Это отношение влияет на температуру Нееля. Зная общую зависимость поля обменного смещения от температуры, легко пересчитать его для других значений $R$. Обменный интеграл на границе соприкосновения пленок $R_{E B}=1.0$. Как было показано в работе [6] поле обменного смещения прямо пропорционально $R_{E B}$. Определение поля обменного смещения при одном значении $R_{E B}$ позволяет вычислить его при остальных значениях.

Для введенных параметров были вычислены температура Нееля $T_{N}=2.71$ и температуры Кюри $T_{C}=3.83$. Поле обменного подмагничивания проявляется при возникновении антиферромагнитного упорядочивания. Исследования проводились для температуры от $T=1.5$ до $T=3.3$ с шагом $\Delta T=0.1$.

На Рис. 1 представлено смещение петли гистерезиса при понижении температуры системы ниже температуры Нееля.

Как видно из Рис. 1 при понижении температуры происходит смещение петли гистерезиса в направлении индукции магнитного поля. При этом увеличивается ширина петли гистерезиса. Для каждой температуры определялось значение поля обменного смещения $h_{E B}$ и ширина петли гистерезиса $D_{h}$. На Рис. 2 представлена зависимость поля обменного смещения $h_{E B}$ от температуры системы $T$.

Как видно из графика на Рис. 2 поле обменного подмагничивания появляется при температуре немного ниже температуры Нееля за счет возникновения областей упорядоченных спинов на границе соприкосновения пленки с подложкой. При понижении температуры от температуры Нееля $\left(T_{N}=2.7\right)$ до температуры $T_{1}=2.0$ поле обменного подмагничивания растет по линейному закону. При температурах ниже $T_{1}$ поле обменного подмагничивания выходит на насыщение. Наличие этих двух границ линейного закона может быть объяснено из анализа зависимости параметра порядка антиферромагнитной подложки от температуры (Рис. 3). При температуре ниже температуры Нееля начинается резкий рост параметра порядка. При этом растет намагниченность слоя граничного с ферромагнитной пленкой, что приводит к существенному влиянию антиферромагнитной подложки на упорядочивание спинов в ферромагнитной пленке. При температуре $T_{1}$ шахматная намагниченность подложки выходит на насыщение. При этом поверхностный слой также выходит на насыщение и становится почти полностью скомпенсированным.

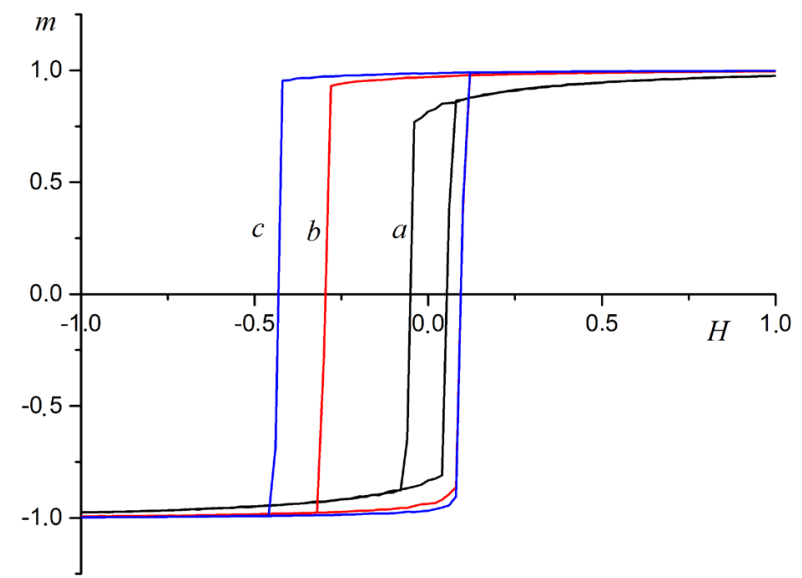

Puc. 1. (Color online) Петля гистерезиса при различных температурах ( $L=36): T=3.2$ (a), $T=2.4$ (b), $T=2.1$ (c).

Fig. 1. (Color online) Hysteresis loop at different temperatures $(L=36): T=3.2(\mathrm{a}), T=2.4$ (b), $T=2.1$ (c).

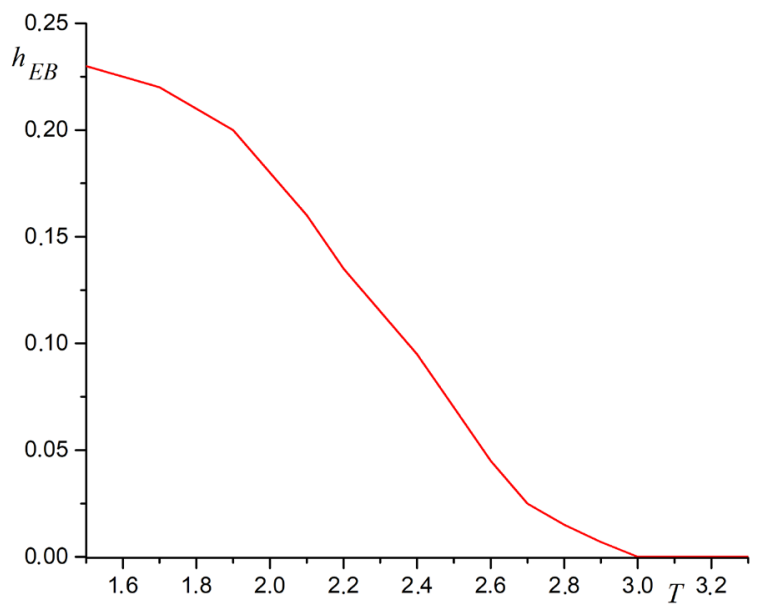

Рис. 2. Зависимость поля обменного смещения $h_{E B}$ от температуры $T(L=36)$.

Fig. 2. The dependence of the exchange bias $h_{E B}$ on the temperature $T(L=36)$.

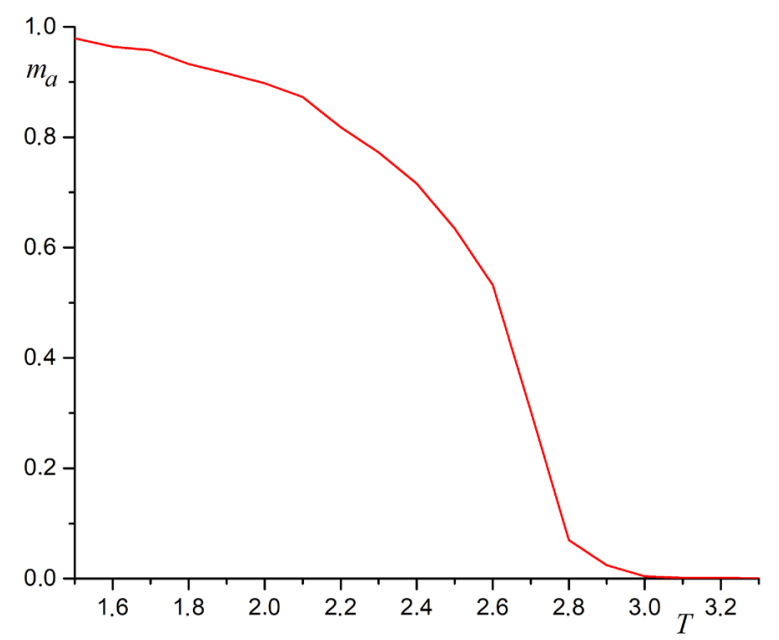

Рис. 3. Зависимость шахматной намагниченности подложки $m_{a}$ от температуры $T(L=36)$.

Fig. 3. The dependence of the chess magnetization for the substrate $m_{a}$ on the temperature $T(L=36)$. 
Исходя из Рис. 2 можно сделать вывод о линейной зависимости поля обменного подмагничивания от температуры в интервале температур от $T_{1}=2.0$ до $T_{N}=2.7$. Объединяя полученные результаты с выводами из работы [6] можем записать:

$$
h_{E B} \sim \frac{J_{E B}}{D_{F M}}(2.7-T) .
$$

Этот результат находится в хорошем согласии с теоретическими предсказаниями [22], согласно которым поле обменного смещения изменяется по закону близкому к линейному.

$$
h_{E B} \sim\left(1-\frac{T}{T_{N}}\right)^{n} \text {, }
$$

где $n \geq 1$.

На Рис. 4 изображена зависимость ширины петли гистерезиса $D_{h}$ от температуры $T$. Аппроксимация результатов компьютерного моделирования показывает, что в согласии с экспериментальными данными ширина петли гистерезиса растет с понижением температуры системы. Зависимость ширины петли гистерезиса $D_{h}$ от температуры T носит характер близкий к линейному. Аппроксимация значений компьютерного эксперимента приводит к следующей зависимости:

$$
D_{h}=(-0.41 \pm 0.02) T+(1.38 \pm 0.04) \text {. }
$$

Увеличение ширины петли гистерезиса связано с влиянием обменного взаимодействия на интерфейсе ферромагнетика и антиферромагнетика, препятствующим изменению направления спинов, а значит и намагниченности. Этот эффект активно используется в устройствах спинтроники для закрепления направления намагниченности ферромагнитных пленок.

\section{4. Заключение}

Результаты компьютерного моделирования показывают, что температура бислойной системы, состоящей из ферромагнитной пленки на антиферромагнитной подложке, оказывает существенное влияние на величину поля обменного подмагничивания на интерфейсе пленок. Это влияние обусловлено в основном состоянием антиферромагнетика. Изменение состояния ферромагнетика можно не учитывать, так как система изучалась при температурах существенно ниже температуры Кюри. При этом отличие от температуры Нееля было не таким значительным. В результате слой спинов антиферромагнетика на интерфейсе с ферромагнетиком был не полностью ориентирован в одну сторону, что снижало влияние антиферромагнетика. Влияние слоя антиферромагнетика на намагниченность ферромагнитной пленки приводит как к изменению положения петли гистерезиса, так и ее ширины.

Эти эффекты наблюдались экспериментально в большом количестве работ. Наибольшие значения поля обменного смещения были получены для пленок $\mathrm{CoFe} / \mathrm{MnN}$ $h_{E B}=1800$ Э для тонкого слоя CoFe толщиной 1.6 нм [23].

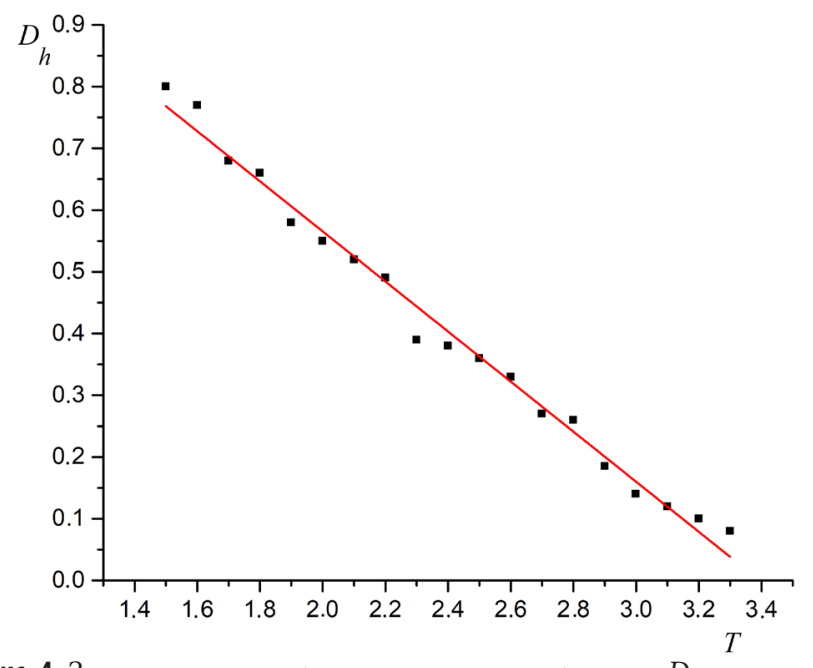

Рис. 4. Зависимость ширины петли гистерезиса $D_{h}$ от температуры $T(L=36)$.

Fig. 4. Dependence of hysteresis loop width $D_{h}$ on temperature $T$ $(L=36)$.

Для ультратонкого тонкого слоя $\mathrm{CoFeB}$ толщиной 0.65 нм в системе $\mathrm{MnN} / \mathrm{CoFeB} / \mathrm{MgO} / \mathrm{TA}$ может быть достигнуто значение $h_{E B}=3600$ Э [24].

Экспериментальное исследование зависимости обменного смещения от температуры, проведенное в работе [25], показало, что для системы $\mathrm{GdBaCo}_{2} \mathrm{O}_{5.5}$ значения параметров имеет следующее значение $t_{C}=275 \pm 2 \mathrm{~K}$, $t_{N}=227 \pm 8 \mathrm{~K}, J_{E B}=0.6$ мэВ. При температуре $t=150 \mathrm{~K}$ поле обменного смещения имеет значение $H_{E B}=48 \pm 1$ Э. Пересчет этих значений в относительные значения дает $h_{E B}=0.24$ при $T=1.7$, что согласуется с результатами нашего моделирования, которое приводит к значению $h_{E B}=0.22$ при той же температуре.

Благодарности / Acknowledgements. Исследование выполнено при финансовой поддержке РФФИ в рамках научного проекта № 20-07-00053. / The research was carried out with the financial support of the Russian Foundation for Basic Research within the framework of the scientific project No. 20-07-00053.

\section{Литература/References}

1. J. Nogues, J. Sort, V. Langlais, V. Skumryev, S. Surinach, J.S. Munoz, M.D. Baro. Physics Reports. 422 (3), 65 (2005). Crossref

2. J. Nogues, I. K. Schuller. J. Magn. Magn. Mater. 192, 203 (1999). Crossref

3. M. Kiwi. J. Magn. Magn. Mater. 234, 584 (2001). Crossref

4. A.E. Berkowitz, T. Kentaro. J. Magn. Magn. Mater. 200, 552 (1999). Crossref

5. K. Takano, R.H. Kodama, A.E. Berkowitz, W. Cao, G. Thomas. Phys. Rev. Lett. 79, 1130 (1997). Crossref

6. W.H. Meiklejohn. J. Appl. Phys. 33 (3), 1328 (1962). $\underline{\text { Crossref }}$

7. D. Mauri, H.C. Siegmann, P.S. Bagus, E. Kay. J. Appl. Phys. 62 (7), 3047 (1988). 당s

8. G. Scholten, K. Usadel, U. Nowak. Physical Review B. 71 (6), 1 (2005). $\underline{\text { Crossref }}$ 
9. H. Ohldag, H. Shi, E. Arenholz, J. Stohr, D. Lederman. Physical Review Letters. 96 (2), 1 (2006). Crossref

10. D. Suess, M. Kirschner, T. Schrefl, J. Fidler, R. L. Stamps, J.-V. Kim. Phys. Rev. B. 67, 054419 (2003). Crossref

11. O. Billoni, A. Tamarit, S. Cannas. Physica B. 384, 184 (2006). Crossref

12. J. Spray, U. Nowak. Journal of Physics D: Applied Physics. 39, 4536 (2006). Crossref

13. Y. Sakurai, H. Fujiwara. J. Appl. Phys. 93 (10), 8615 (2003). Crossref

14. J.-V. Kim, R. L. Stamps. Phys. Rev. B. 71, 094405 (2005). Crossref

15. S. V. Belim. Letters on Materials. 10 (3), 272 (2020). (in Russian) [С.В. Белим. Письма о материалах. 10 (3), 272 (2020).] Crossref

16. S. V. Belim, S.S. Belim. Journal of Physics: Conference Series. 1697, 012098 (2020). Crossref
17. S. V. Belim, I. B. Larionov. Journal of Physics: Conference Series. 1546, 012111 (2020). Crossref

18. O. V. Billoni, S. A. Cannas, F. A. Tamarit. J. Phys.: Condens. Matter. 23, 386004 (2011). Crossref

19. D. P. Landau, K. Binder. Phys. Rev. B. 17, 2328 (1978). Crossref

20. U. Wolff. Physical Review Letters. 62, 361 (1988). Crossref

21. J.S. Wang, R. H. Swendsen. Physica A. 167, 565 (1990). Crossref

22. S. K. Giri and T.K. Nath, J. Nanosci. Nanotechnol. 14, 1209 (2014). Crossref

23. M. Meinert, B. Buker, D. Graulich, M. Dunz. Phys. Rev. B. 92, 144408 (2015). Crossref

24. P. Zilske, D. Graulich, M. Dunz, M. Meinert. Appl. Phys. Lett. 110, 192402 (2017). Crossref

25. N. I. Solin, S. V. Naumov, S. V. Telegin et al. J. Exp. Theor. Phys. 125, 1096 (2017). Crossref 